\title{
Processing of Internal and External Signals for Performance Monitoring in the Context of Emotional Faces
}

\author{
Christian Valt, Marina Palazova, and Birgit Stürmer
}

International Psychoanalytic University, Berlin

ABSTRACT

Performance monitoring can be based on internal or external signals. We recorded event-related potentials (ERPs) to investigate whether relating performance to external signals affects internal performance monitoring. Thirty participants performed a task in which responses were followed by faces whose expressions were partially contingent upon performance. Instructions given to half of the participants mentioned a link between task performance and the upcoming face expression. Instructed participants showed smaller error-related negativity (Ne/ERN) to erroneous responses and larger N170 to faces as compared to participants in the not-instructed group. In addition, we observed a correlation between $\triangle \mathrm{Ne}$ /ERN and P1-latency benefit for angry faces after errors. Taken together, processing of internally generated signals for performance monitoring is reduced by instructions referring to an emotional face. Furthermore, we relate the correlation between the magnitude of internal monitoring and facilitation in processing angry faces to priming induced by the negative affective meaning of errors.

performance monitoring emotional face expressions, error-related negativity (Ne/ERN), early visually-evoked potentials, N170

\section{INTRODUCTION}

We constantly evaluate our actions according to the goal we aim for. Performance monitoring is, therefore, one of the major demands in human information processing. Performance monitoring can be based on internal signals generated by the person itself or external signals from the environment.

In unambiguous situations, we generally detect our errors based on internally generated signals. In these cases, an error is detected whenever new evidence that supports the correct response accumulates after the incorrect response was made (Yeung \& Summerfield, 2012). In effect, errors are often followed by quick corrections (Rabbitt, 1966) as well as slower and more accurate responses to the successive trial (Gehring, Goss, Coles, Meyer, \& Donchin, 1993; Themanson, Rosen, Pontifex, Hillman, \& McAuley, 2012). In many situations, external signals are also important sources of information for performance monitoring. Among the variety of external signals present in the environment, facial expressions are important means of evaluating the appropriateness of our behaviour (Blair, 2003). The inclination of infants to attend to caregivers' emotional facial expressions in various situations is clear evidence of the developmental relevance of this external signal (Klinnert, Campos, Sorce, Emde, \& Svejda, 1983). For example, infants adapt their preferences toward objects according to the emotion expressed by an experimenter (Moses, Baldwin, Rosicky, \& Tidball, 2001; Repacholi, 1998) or undertake a specific behaviour based on the facial emotion of the caregiver (Sorce, Emde, Campos, \& Klinnert, 1985).

With the present study, we aimed to investigate the interplay of internal and external sources for performance monitoring. Participants performed a choice-response task on Eriksen-Simon stimuli (Eriksen \& Eriksen, 1974; Simon, 1969) and emotional face expressions were used as external signals after the participants' responses. Faces were mainly angry after wrong responses and neutral after correct responses. We analysed event-related brain potentials (ERPs) to test changes in information processing when instructions suggested a focus on internal or external signals.

A negative deflection over the midline fronto-central electrodes peaking $50-100 \mathrm{~ms}$ after response execution is seen as a manifesta-

Corresponding author: Dr. Christian Valt, International Psychoanalytic University, Stromstrasse, 3b, Berlin, 10555 Germany. Email: christian.valt@ ipu-berlin.de 
tion of internal performance monitoring (Falkenstein, Hohnsbein, Hoormann, \& Blanke, 1991; Gehring et al., 1993). This response-related negativity is more robust after errors ( $\mathrm{Ne}$, or error-related negativity, ERN) than after correct responses (correct-related negativity, CRN). Apart from the Ne/ERN and the CRN, a second ERP component is related to internal performance evaluation. This component is called error positivity (Pe, Falkenstein et al., 1991; and Pc, for the positivity after correct responses) and has been associated with conscious errormonitoring (Shalgi, Barkan, \& Deouell, 2009), evidence accumulation (Steinhauser \& Yeung, 2012), or confidence evaluation (Boldt \& Yeung, 2015). A study using principal component analysis reported that the Pe consists of two subcomponents: an early fronto-central component that immediately follows the Ne/ERN and a late centro-parietal component, peaking 200-400 ms after the response (Arbel \& Donchin, 2009).

The most prominent ERP component concerning the processing of external signals for performance monitoring is the so-called feedbackrelated negativity (FRN) - an ERP component elicited around $250 \mathrm{~ms}$ after the onset of informative feedback stimuli (Nieuwenhuis, Yeung, Holroyd, Schurger, \& Cohen, 2004). This potential is generally evoked when internal signals fail in an accurate performance evaluation because of low sensitivity (Heldmann, Rüsseler, \& Münte, 2008; Stahl, 2010) or uncertainty of the stimulus-response association (Holroyd \& Coles, 2002; Nieuwenhuis et al., 2002), and it is influenced by goal conduciveness (Gentsch, Grandjean, \& Scherer, 2013) and goal relevance (Walentowska, Moors, Paul, \& Pourtois, 2016). Notably, when faces are used as feedback stimuli, modulations of both the FRN and early visual ERP components of face processing are also observed (Aarts \& Pourtois, 2012).

In summary, components in the response-locked ERP reflect processing of internal signals for performance monitoring. Evaluation of external signals, instead, is mainly evident as FRN, but modulations of early visually evoked potentials are also expected when the feedback stimulus is an emotional facial expression.

Interest in contextual and goal-related factors on the dynamics of signal processing for performance monitoring has recently grown (Ullsperger, Danielmeier, \& Jocham, 2014). Two highly relevant contextual factors for signal processing are (a) how certain one could be about the stimulus-response mapping and (b) the feedback. In the absence of an established stimulus-response mapping, as in probabilistic learning, informative feedback results in increased internal signal processing and reduced external signal processing in the course of learning (Bellebaum \& Colosio, 2014; Holroyd \& Coles, 2002; Müller, Möller, Rodriguez-Fornells, \& Münte, 2005). External feedback signals are preferentially processed, instead, when the stimulus-response mapping is difficult to learn, as with random feedback (Holroyd \& Coles, 2002). When instructions clearly state the stimulus-response mapping, performance monitoring is mostly based on internal signals and the external feedback signals are processed in case internal signals fail a precise performance evaluation, as for response speed monitoring (Heldmann et al., 2008; Stahl, 2010). However, Olvet and Hajcak (2009a) showed that with well-established stimulus-response mappings, a feedback related to performance accuracy did not affect the Ne/ERN, probably because feedback is redundant (Boksem, Ruys, \& Aarts, 2011). The FRN is, however, enhanced in case of ambiguous feedback (Gibbons, Schnuerch, \& Stahl, 2016). Furthermore, Ogawa, Masaki, Yamazaki, and Sommer (2011) reported reductions of the Ne/ERN amplitude when a negative admonishment is delivered as feedback.

Moreover, external signals seem to acquire a special relevance when they are informative about performance speed. Walentowska et al. (2016) reported that the FRN was modulated when feedback was related to response speed whereas the CRN was modulated with random feedback. Stahl (2010) showed that responses that were difficult to categorise according to response speed were associated with a smaller CRN and a larger FRN compared to responses that were easily categorised, because of being clearly faster or slower than a deadline (see also Heldmann et al., 2008). Taken together, internal and external signal processing for performance monitoring is flexible and depends on feedback content and person's certainty about performance quality.

With the present experiment, we investigated potential changes in the processing of internal and external signals for performance monitoring depending on instructions. In other words, we explored whether an explicit instruction about a relationship between one's own performance and an external stimulus is sufficient to reduce internal monitoring in favour of the processing of an external signal. Half of the participants (instructed group) were instructed that the external signals were linked to their performance, whereas for the other half of the participants (not-instructed group), this relationship was not mentioned in the instructions given before the experiment. Importantly, the relationship between feedback and performance was identical for all participants, irrespective of their instructions, and participants in both groups received clear information about the stimulus-response mapping. Many studies have shown that instructions strongly affect the processing of internal signals when they invite the participant to give priority to either speed or accuracy, affecting in this way also the importance of errors (Gehring et al., 1993). In the present experiment, instead, accuracy and speed were equally important in both groups. By manipulating just the instructions between groups, without changing the feedback contingency on performance, we aimed to obtain two different monitoring conditions: one that promotes the monitoring of both internal and external signals (instructed group) and one in which internal signals should be preferentially monitored (not-instructed group). Emotional faces were used as external stimuli in order to create a context with social connotations between performance and external stimulus: A neutral face generally followed a correct response while an angry face usually followed an error. In real life, we constantly monitor the reactions of people interacting with us as feedback on our own behaviour. Therefore, the facial expressions of emotion might be an ecologically important external signal for performance monitoring.

With the employed experimental manipulation, the Ne/ERN and later response-locked components might be altered with respect to the focus of performance monitoring. That is, internal signals of performance monitoring might be reduced when attention is directed by instructions towards an external signal. On the contrary, the FRN should 
be enhanced in the instructed compared to the not-instructed group. In addition, it is of particular interest whether directing the focus of performance monitoring towards an external signal would also affect early processing of the signal itself. Allocating attention to a stimulus generally increases the amplitude of early visual potentials (Hillyard \& Anllo-Vento, 1998; Mangun \& Hillyard, 1991): In face processing, the amplitude of the N170 is strongly enhanced by attentional allocation to faces (Holmes, Vuilleumier, \& Eimer, 2003). Therefore, early ERP components like the P1 and the N170 might be enhanced when instructions induce an external focus for performance monitoring.

The present design also allows testing whether errors prime the processing of emotional faces, as suggested for words by Aarts, De Houwer, and Pourtois $(2012,2013)$. They showed that the amplitude difference between the $\mathrm{Ne} / \mathrm{ERN}$ and the CRN $(\triangle \mathrm{Ne} / \mathrm{ERN})$ correlated with behavioural facilitation, evident as faster valence judgement, when the emotional meaning of a word was congruent to the negative or the positive connotation of incorrect and correct responses, respectively. Here, affective priming should lead to faster processing of angry faces after errors than after correct responses. A correlation between the $\triangle \mathrm{Ne} / \mathrm{ERN}$ and the P1-latency benefit for angry faces after errors might corroborate affective priming.

\section{MATERIALS AND METHODS}

\section{Participants}

Thirty young adults $\left(M_{\text {age }}=25\right.$ years, range: $19-32$ years, 20 women) participated in return for a $€ 20$ reimbursement. All participants had normal or corrected-to-normal vision. According to the Edinburgh Handedness Inventory (Oldfield, 1971), all participants were righthanded $(M=79.8 \%)$, except for two ambidextrous participants. All participants gave written informed consent according to the Declaration of Helsinki.

\section{TABLE 1.}

Demographic and personality description of the participants in the two experimental groups

\begin{tabular}{cccc}
\hline & Instructed & Not-instructed & \\
\hline Sex & 5 males & 5 males & \\
Age & 25 years (19-32) & 26 years (21-31) & \\
\hline BIS & Instructed & Not-instructed & \\
BAS & $21.20(0.76)$ & $19.73(1.13)$ & $n s$ \\
Drive & $39.13(0.74)$ & $39.80(0.96)$ & $n s$ \\
Fun Seeking & $11.60(0.36)$ & $11.07(0.61)$ & $n s$ \\
Reward & $11.67(0.42)$ & $12.20(0.45)$ & $n s$ \\
Responsiveness & $15.87(0.47)$ & $16.53(0.54)$ & $n s$ \\
\hline
\end{tabular}

Note. The reported values represent means (standard errors). BIS: Behavioral Inhibition System; BAS: Behavioral Approach System; $n s: p$ value $>.05$.
Participants were randomly divided in two groups (instructed, not-instructed). At the end of the experiment, all participants were administered the German version of the Behavioral Inhibition System/ Behavioral Approach System (BIS/BAS) scale (Carver \& White, 1994) in order to control for possible between-groups differences in the sensitivity to punishment and reward (see Table 1).

\section{Stimuli, Design, and Procedure}

A combination of an Eriksen flanker task (Eriksen \& Eriksen, 1974) and a Simon task (Simon, 1969) was the response-choice task in the present study. A $3 \times 3$ array of the letters $M, N, W$, or $H$ constituted the stimulus for the choice-response task. Each array was made up of a frame of flanker letters and a central target letter that could be either identical to or different from the surrounding letters (see Figure 1); letters appeared as targets or as flankers with an equal probability (i.e., $25 \%$ as target, $25 \%$ as flanker). The stimulus array was presented above or below a central fixation cross (centre-to-centre distance: $0.80^{\circ}$ of visual angle). The parametric manipulation of two stimulus positions, four flanker and four target letters, generated 32 stimuli, presented once in each experimental block. Single letters and the fixation cross had each a size of $0.32^{\circ} \times 0.32^{\circ}$ and were separated by a gap of $0.05^{\circ}$. The letters and the fixation cross had a dark grey colour (RGB: 78, 78, 78 ) and were displayed on a light grey background (RGB: 128, 128, 128).

Participants were requested to respond to the identity of the central target letter by pressing with their left and right index fingers one of two response buttons arranged vertically on the desk (see Figure 1). Two letters required a response with the upper button (e.g., the letters $M$ and $N$ ) and the other two letters required a response with the lower button (e.g., the letters $H$ and $W$ ). The mapping of target letters to the response buttons was counterbalanced across participants.

Neutral and angry faces of unknown identities were used as face stimuli presented after the response: Eight hundred neutral faces were obtained from various databases available at Humboldt-Universität zu

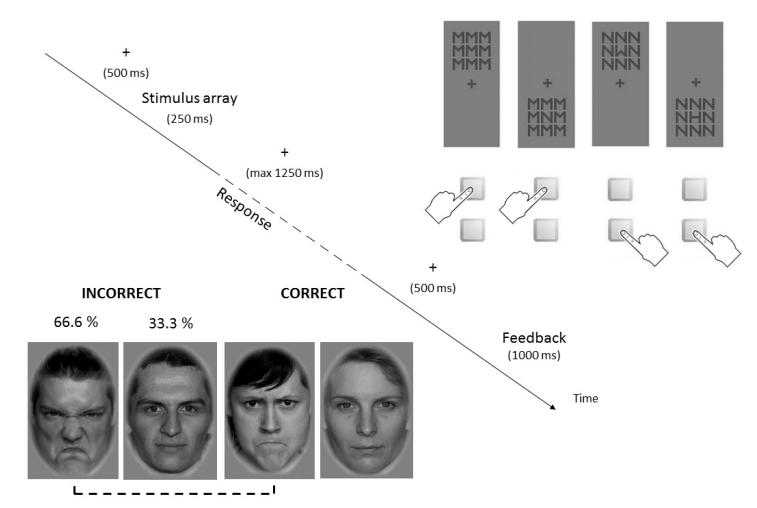

FIGURE 1.

Schematic representation of the response-choice task, the time course of trials, and the faces used as performance feedback. 
Berlin; a hundred-sixty angry faces were selected from the FACES database (Ebner, Riediger, \& Lindenberger, 2010). Faces were converted to greyscale and fitted into a $6.81^{\circ} \times 4.52^{\circ}$ vertical ellipse; the colour thereof matched the background colour. All the faces were normalised on the dimensions position, size, and luminance contrast. From the neutral faces, 775 faces were used for the experimental trials and the remaining 25 faces were selected for a recognition task.

Each trial started with the presentation of the stimulus array, displayed for $250 \mathrm{~ms}$, followed by a fixation cross displayed for a maximum duration of 1,250 ms (see Figure 1). Participants had $1.5 \mathrm{~s}$ to respond. Directly after the response, the fixation cross was displayed for further $500 \mathrm{~ms}$. Then, a face was presented for $1 \mathrm{~s}$ at the centre of the screen. After the face offset, the fixation cross was presented again for $500 \mathrm{~ms}$ before the next trial started with the presentation of a new stimulus array.

Participants practised the response-choice task in three practice blocks of 32 trials each. In case the participant committed too many errors (more than 10) in the last two practice blocks, two additional practice blocks were performed before the beginning of the experiment and the participant was encouraged to be more accurate. During the practice blocks, incorrect responses were signalled by the fixation cross turning red for $500 \mathrm{~ms}$. The colour of the fixation cross did not change when the participant gave a correct response. Faces were not displayed during the practice blocks.

After the practice blocks, directly before the start of the main experiment, all participants were instructed that each response was followed by a face. Half of the participants received an additional piece of information about a relationship between the emotion expressed by the face and their performance in the response-choice task. The instruction given to participants in the instructed group was "a face, whose expression is related to your performance in the task, will be presented after each response"; the instruction given to participants in the notinstructed group was "a face will be presented after each response."

The main experiment consisted of 25 experimental blocks, each with 32 trials in random order. In addition, one to three recognition trials were presented in each block to ensure that participants paid attention to the face stimuli. Faces were displayed after each trial in all the experimental blocks.

Expression of faces following the responses in the experimental blocks was determined by an algorithm that produced an angry face after $66.6 \%$ of incorrect responses and a neutral face in the remaining $33.3 \%$ of incorrect responses (see Figure 1). Presentation of an angry face after an error triggered the presentation of another angry face after a correct response in one of the next 24 trials, allowing an equal number of angry faces after incorrect and correct responses throughout the experiment. All other correct responses, and miss responses, were followed by a neutral face expression.

In recognition trials, a face followed a mask displayed for $500 \mathrm{~ms}$ and the participant had to judge whether the face was identical or different to the one presented in the preceding trial. This was performed as an attention-check to ensure that participants in both groups processed the face stimuli. Half of the faces presented in the recognition task were repeated from the preceding trial, while half were faces never presented before. The $Y$ and $N$ buttons on a German keyboard were used in this task to express a positive or a negative answer; no time limit was imposed for responding. In case of an error in recognition, a feedback recommended the participant to pay more attention to the face stimuli.

To obtain an error rate of $10 \%$ throughout the experiment, written feedback of the average response time (RT) was displayed at the end of each third block (except the second to last block). In case the participant committed fewer than four errors in the preceding three blocks, the feedback encouraged the participant to be faster. In case of more than 15 errors in the last three blocks, the participant was asked to respond more accurately. Additionally, written within-block feedback suggested the participant to be more accurate if five errors were committed in the preceding 10 trials.

In order to control for between-groups personality differences, participants filled out the BIS/BAS (Carver \& White, 1994) scale at the end of the experiment. In fact, a positive correlation between the individual punishment and reward sensitivity and the magnitude of the Ne/ERN has been shown in an experimental context of punishment and reward omission (Boksem, Tops, Kostermans, \& De Cremer, 2008). Participants also answered the question whether they had noticed any relationship between their performance and the emotion expressed by the faces and, in case they did, they had to describe the relationship.

\section{Electrophysiological Recording and Event-Related Potential Calculation}

The electroencephalogram (EEG) was recorded during the whole experiment from $28 \mathrm{Ag} / \mathrm{AgCl}$ electrodes embedded in an elastic cap (Easy-Cap ${ }^{\mathrm{m}}$, EASYCAP GmbH, Herrsching, Germany; URL http:// www.easycap.de). The locations of electrodes were based on the International 10-20 system and corresponded to the positions: Fp1/2, F7/8, F3/4, Fz, FC3/4, FCz, T7/8, C3/4, Cz, CPz, P7/8, P3/4, Pz, $\mathrm{PO} / 10, \mathrm{PO} / / 8, \mathrm{O} 1 / 2$, and $\mathrm{Oz}$. Two electrodes were applied directly on the skin over the left and right mastoids, M1 and M2. Initial common reference was $\mathrm{M} 1$ and $\mathrm{AFz}$ served as ground. The electrooculogram (EOG) was recorded from three electrodes, one placed below the right eye (VEOG) and two on the outer canthi of both eyes (HEOG). All signals were digitised with a frequency of $250 \mathrm{~Hz}$ and a band-pass of 0.05 to $70 \mathrm{~Hz}$. The electrodes' impedance was kept below $5 \mathrm{k} \Omega$ for EEG and below $10 \mathrm{k} \Omega$ for EOG.

Offline, the influence of blinks and eye movements on the EEG was corrected by independent component analysis based on 20 calibration trials for each artifact (left, right, up, down, and blink) obtained after the experiment proper. A band-pass filter with cut-off frequencies of $0.1 \mathrm{~Hz}$ and $30 \mathrm{~Hz}$ and a slope of $48 \mathrm{~dB} /$ octave was applied.

Response-locked epochs started $200 \mathrm{~ms}$ pre-response and lasted until $600 \mathrm{~ms}$ post-response. Stimulus-locked epochs started $200 \mathrm{~ms}$ before the face stimulus was displayed and lasted for $1 \mathrm{~s}$ after the onset of the stimulus. The $200 \mathrm{~ms}$ pre-response and pre-stimulus intervals were used as baseline. Trials were first automatically checked for artifacts (maximum allowed voltage step of $50 \mu \mathrm{V} / \mathrm{ms}$, maximum allowed absolute difference of $200 \mu \mathrm{V}$ in a $200 \mathrm{~ms}$ interval, minimal and maximal 
allowed amplitude of $-200 \mu \mathrm{V}$ and $200 \mu \mathrm{V}$, lowest allowed activity of $0.5 \mu \mathrm{V} / \mathrm{ms}$ in a $100 \mathrm{~ms}$ interval) and then visually inspected. Trials with artifacts were discarded from further analyses. All ERPs were recalculated to average reference based on the electrodes Fp1/2, F7/8, F3/4, Fz, FCz, M1/2, T7/8, C3/4, Cz, CPz, P7/8, P3/4, Pz, and O1/2.

Response-locked epochs were averaged separately according to the correctness of the response in order to obtain response-locked ERPs for correct and incorrect responses. The CRN and the Ne/ERN were calculated as mean amplitudes at the medial fronto-central electrodes between 0 and $100 \mathrm{~ms}$ after response. Early and late Pc and Pe were calculated as the mean amplitude at the medial fronto-central electrodes in the time-window of 150-300 ms (Arbel \& Donchin, 2009) and as the mean amplitude at the medial parietal electrodes in the timewindow of 200-400 ms (Olvet \& Hajcak, 2009b; Pontifex et al., 2010), respectively. The electrodes showing the largest effect of group were then selected for statistical analysis based on visual inspection. The amplitudes of these negative and positive deflections at the selected electrodes were entered into separate repeated-measures analyses of variance (ANOVAs), with Response Correctness (correct responses vs. incorrect responses) as a within-subject factor and Group (instructed vs. not-instructed) as a between-subjects factor. To explore group effects, follow-up independent-samples two-tailed $t$ tests with Group as a between-subjects factor were run. The significance level of $\alpha=.05$ was adjusted according to Bonferroni's correction for multiple pairwise tests $(\alpha / 2=.025)$.

Face-locked epochs were averaged separately according to response correctness and expression of the face. The P1 and the N170 were extracted from the face-locked ERP waves as the positive and the negative peak over left and right parieto-occipital electrodes between 50 and $150 \mathrm{~ms}$ and between 100 and $200 \mathrm{~ms}$, respectively. The FRN was calculated as the mean amplitude of the face-locked ERP at the medial fronto-central electrodes between 210 and $310 \mathrm{~ms}$ after onset of the face stimulus (Nieuwenhuis et al., 2004). The electrodes showing the largest effect of group were then selected for statistical analysis based on visual inspection. The activity of the FRN and the amplitude and latency of the P1 and the N170 peaks at the selected electrodes were entered into separate repeated-measures ANOVAs with Response Correctness (correct response vs. incorrect response) and Emotion (neutral vs. angry) as within-subject factors and Group (instructed vs. not-instructed) as a between-subjects factor. Follow-up related-samples two-tailed $t$ tests were run to explore the meaning of significant interactions. The significance level of $\alpha=.05$ was adjusted according to Bonferroni's correction for multiple pairwise tests $(\alpha / 4=.0125)$.

\section{RESULTS}

Mann-Whitney's $U$ tests for independent-samples performed on the BIS/BAS scores indicated that the two groups did not significantly differ neither in the BIS-scale score, $U=82.5, p=.217$, nor in any of the BAS-subscale scores, $U s>81.5$, $p s>.202$ (see Table 1 ).

At the end of the experiment, 19 participants correctly reported the general relationship between angry faces and errors. Six participants in the instructed group and five participants in the not-instructed group did not notice any relation.

\section{Behavioural Performance}

Mean error rate in the response-choice task was $8.79 \%(S E=0.85 \%)$ and ranged from $2.38 \%$ to $24.50 \%$ across participants. Group did not significantly affect participants' mean error rate $(t[28]<1.00$; instructed: $M=9.41 \%, S E=1.57 \%$; not-instructed: $M=8.18 \%, S E=0.67 \%$ ) and RTs for correct $(t[28]<1.00$; instructed: $M=743 \mathrm{~ms}, S E=18 \mathrm{~ms}$; not-instructed: $M=723 \mathrm{~ms}, S E=19 \mathrm{~ms})$ or incorrect $(t[28]<1.00$; instructed: $M=714 \mathrm{~ms}, S E=28 \mathrm{~ms}$; not-instructed: $M=706 \mathrm{~ms}, S E=$ $29 \mathrm{~ms}$ ) responses.

Accuracy in the recognition task was $91.38 \%(S E=3.16 \%)$ for the instructed group and $95.71 \%(S E=0.88 \%)$ for the not-instructed group. Recognition performances in the two groups did not differ significantly, $t(28)=1.32, p=.20, r=.24$.

\section{Processing of Internal Signals}

The average number of trials for the ERP calculation of incorrect and correct responses was $66.2(S E=5.1$, range: 19-121) and $721.5(S E=$ 7.1, range: 601-778), respectively. The amplitude of the negative deflection at electrode $\mathrm{Fz}$ was used to investigate the Ne/ERN and CRN activity in the two groups; the activities at electrode $\mathrm{FCz}$ and at electrode $\mathrm{Pz}$ were used to explore effects of instructions on the early and the late positivity.

The ANOVA performed on activity at electrodes $\mathrm{Fz}$ showed a significant effect of response correctness, $F(1,28)=56.00, p<.001$, $\eta_{p}^{2}=.67$, indicating larger negativity after errors (Ne/ERN) than after correct responses (CRN), but no main effect of group, $F(1,28)=$ $1.55, p=.22, \eta_{\mathrm{p}}^{2}=.05$. Importantly, group significantly interacted with response correctness, $F(1,28)=8.87, p=.006, \eta_{p}^{2}=.24$, indicating that the difference between $\mathrm{Ne} / \mathrm{ERN}$ and $\mathrm{CRN}(\triangle \mathrm{Ne} / \mathrm{ERN})$ was larger in the not-instructed group than in the instructed group. Follow-up $t$ tests, with adjusted a levels of .025 , showed that group significantly modulated the amplitude of the Ne/ERN, $t(28)=2.73, p=.011, r=.46$, but not the amplitude of the CRN, $t<1.00$. The Ne/ERN in the notinstructed group was more negative than the Ne/ERN in the instructed group (see Figure 2).

The amplitude of the positive deflection at $\mathrm{FCz}$ presented a significantly different amplitude, $F(1,28)=35.22, p<.001, \eta_{p}^{2}=.56$, with larger positivity for the early Pe than the early Pc. Group did not have any significant effect on the amplitude of this positivity $F(1,28)=1.41$, $p=.24, \eta_{\mathrm{p}}^{2}=.05$, and did not significantly interact with response correctness, $F<1.00$. These results suggest that group did not affect the amplitude of the early positive potential and did not affect the difference between early Pc and early Pe (see Figure 2).

A similar pattern of results was obtained for the late positive potential. The difference between late Pc and late Pe was significant, $F(1$, $28)=71.42, p<.001, \eta_{p}^{2}=.72$, but group was not significant as a main effect, $F<1.00$, and did not interact with response correctness, $F<1.00$ (see Figure 2). 


\section{Processing of External Signals}

The average number of trials in the four conditions was 42.4 ( $S E=3.6$, range: $12-80$ ) for angry faces after errors, $42.3(S E=2.6$, range: $12-78)$ for angry faces after correct responses, $27.6(S E=2.6$, range: $10-41)$ for neutral faces after errors, and 658.8 ( $S E=10.6$, range: $520-750)$ for neutral faces after correct responses.

Overall, the P1 and the N170 amplitudes were larger over the parieto-occipital electrodes, with a maximum effect of group at PO10 (see Figure 3). The electrode Fz was used for FRN analyses.

\section{P1 AND N170}

Response correctness had a significant effect on the amplitude of the $\mathrm{P} 1, F(1,28)=21.05, p<.001, \eta_{\mathrm{p}}^{2}=.43$, with a larger P1 after incorrect responses than after correct responses, but the amplitude of the P1 was not significantly affected by emotion, $F(1,28)=1.24, p=.28, \eta_{\mathrm{p}}^{2}=$ .04 (see Figure 3 ). The factor group was not significant in the analysis

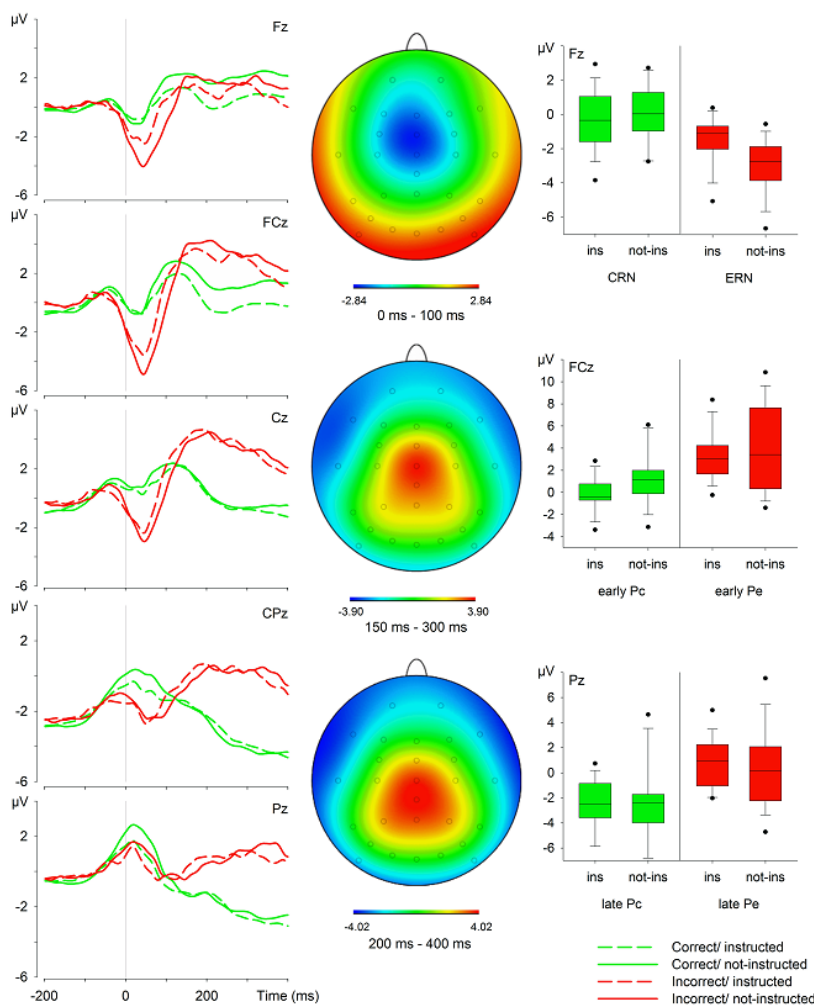

FIGURE 2.

Response-locked grand average ERPs at medial electrodes for correct and incorrect responses in the instructed and the not-instructed group. Topographical maps of the subtracted brain activity in the $\mathrm{Ne} / \mathrm{ERN}-\mathrm{CRN}(0-100 \mathrm{~ms})$, the early Pe-Pc (150-300 ms) and the late Pe-Pc (200-400 ms) timewindows. Box plots of the mean activity in the two groups (ins: instructed vs. not-ins: not-instructed) separately for the CRN and the Ne/ERN, for the early Pc and the early Pe, and for the late Pc and the late Pe. of the P1 amplitude, $F<1.00$. The interaction between emotion and response correctness was not significant, $F(1,28)=2.66, p=.11, \eta_{\mathrm{p}}^{2}$ $=.09$, and group did not interact with any effect on the P1 amplitude, Fs $<1.00$.

The two within-subject factors, emotion and response correctness, had both a significant main effect on P1 latency: emotion, $F(1,28)=$ $48.65, p<.001, \eta_{\mathrm{p}}^{2}=.63$, and response correctness, $F(1,28)=125.19$, $p<.001, \eta_{\mathrm{p}}^{2}=.82$; but, the between-subjects factor did not show a significant main effect, $F<1.00$ (see Figure 3 ). The two main effects of emotion and response correctness were further defined by a significant interaction, $F(1,28)=48.45, p<.001, \eta_{\mathrm{p}}^{2}=.64$. Follow-up comparisons, with adjusted $\alpha$ levels of .0125 , were performed to explore the meaning of the interaction. The latencies of the P1 were slower for angry faces after a correct response $(124 \mathrm{~ms})$ than after an incorrect response (110 $\mathrm{ms}), t(29)=14.39, p<.001, r=.94$. For neutral faces, the P1 latency after a correct response $(123 \mathrm{~ms})$ did not differ from that after an incor-

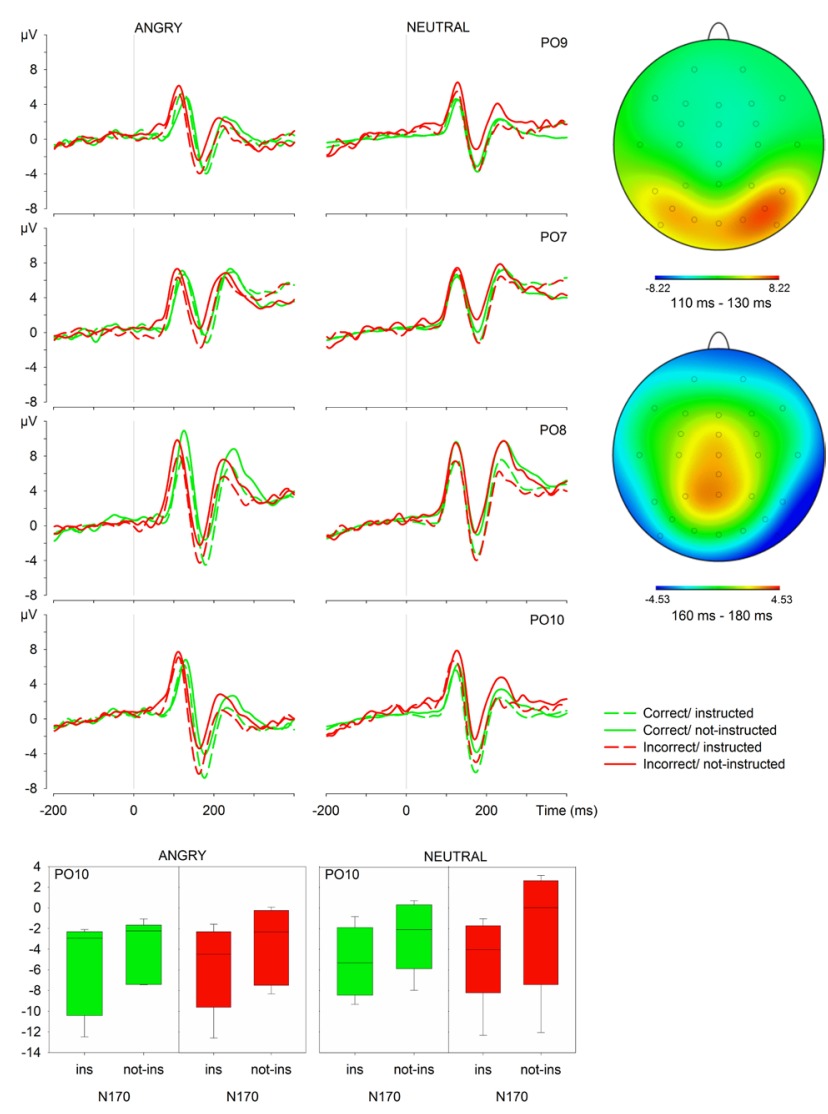

FIGURE 3.

Face-locked grand average ERPs at parieto-occipital electrodes for correct and incorrect trials in the instructed and not-instructed group, separately for angry and neutral faces. Topographical maps of the brain activity in the P1 (110-130 $\mathrm{ms}$ ) and the N170 (160-180 ms) time-windows. Box plots of the mean amplitude of the N170 peak in the two instruction groups (ins: instructed vs. not-ins: not-instructed), separately for angry and neutral faces after correct or incorrect responses. 
rect response (121 ms), $t(29)=1.51, p=.14, r=.27$. Furthermore, emotion had a significant effect on the P1 latency for errors, $t(29)=7.87, p$ $<.001, r=.82$, but not for correct responses, $t(29)<1.00$. The effects of emotion and response correctness were not modulated by group, $F \mathrm{~s}(1$, $28)<3.11$, ps $>.09, \eta_{p}^{2}<.10$, and the three-way interaction also failed to reach significance, $F(1,28)=2.67, p=.11, \eta_{\mathrm{p}}^{2}=.09$.

The N170 amplitude showed a significant main effect of response correctness, $F(1,28)=8.41, p=.007, \eta_{\mathrm{p}}^{2}=.23$, with a larger amplitude for correct than for incorrect responses, and a significant main effect of emotion, $F(1,28)=8.26, p=.008, \eta_{\mathrm{p}}^{2}=.23$, with a more negative $\mathrm{N} 170$ for angry than for neutral faces (see Figure 3). Notably, the betweensubjects main effect of group was significant for N170 amplitude, $F(1,28)=4.18, p=.05, \eta_{\mathrm{p}}^{2}=.13$, indicating larger N170 amplitudes in the instructed group as compared to the not-instructed group. The interactions between factors were all short of significance in the N170 analyses, Fs $<1.00$.

The N170 latency showed a significant main effect of response correctness, $F(1,28)=58.83, p<.001, \eta_{\mathrm{p}}^{2}=.68$, and of emotion, $F(1,28)$ $=10.00, p=.004, \eta_{\mathrm{p}}^{2}=.26$, but no main effect of group, $F<1.00$. These two significant main effects were further defined by a significant interaction, $F(1,28)=26.46, p<.001, \eta_{p}^{2}=.49$. Follow-up comparisons, with adjusted $a$ levels of .0125 , were performed to explore the meaning of the interaction. The N170 for angry faces peaked earlier after an incorrect response $(160 \mathrm{~ms})$ than after a correct response $(174 \mathrm{~ms})$, $t(29)=9.12, p<.001, r=.86$. For neutral faces, the N170 latency after a correct response $(172 \mathrm{~ms})$ did not differ from that after an incorrect response (169 ms), $t(29)=1.77, p=.09, r=.31$. Furthermore, emotion altered the N170 latency after errors, $t(29)=5.34, p<.001, r=.70$, but not after correct responses, $t(29)=1.52, p=.14, r=.27$. No other interaction was significant, $F s<1.00$.

\section{FEEDBACK-RELATED NEGATIVITY}

The FRN component was significantly modulated by the emotion expressed by the face, $F(1,28)=12.43, p<.001, \eta_{\mathrm{p}}^{2}=.30$ : It was smaller

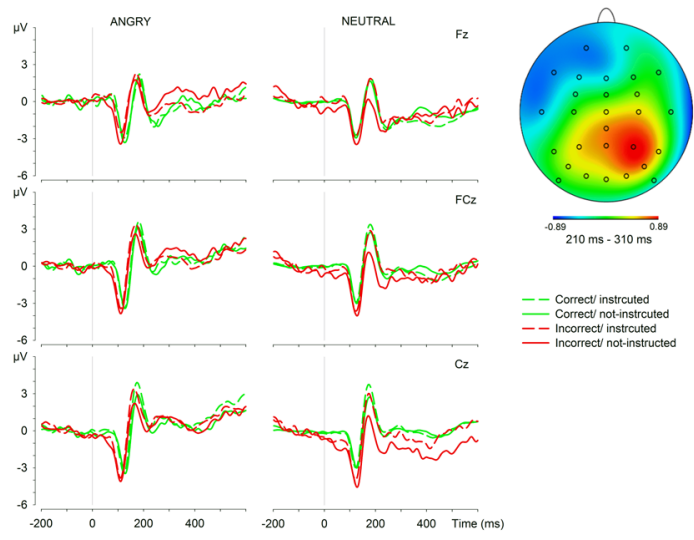

\section{FIGURE 4.}

Face-locked grand average ERPs at medial fronto-central electrodes for correct and incorrect trials in the instructed and not-instructed group, separately for angry and neutral faces. Topographical maps of subtracted brain activity in the FRN (210-310 ms) time-windows. for angry than for neutral faces (see Figure 4). However, the FRN was not modulated by response correctness, $F(1,28)=2.24, p=.15, \eta_{\mathrm{p}}^{2}=$ .07 , and by group, $F<1.00$. All the interactions between factors failed to reach significance, $F \mathrm{~s}(1,28)<2.37$, $p s>.13, \eta_{\mathrm{p}}^{2}<.08$.

\section{Affective Priming}

The P1-latency benefit, computed as the latency difference between the $\mathrm{P} 1$ peak for angry faces presented after errors and the P1 peak for angry faces presented after correct responses, correlated significantly with the amplitude difference between the Ne/ERN and the CRN, $r=.41, p=$ .03. That is, the larger the $\Delta \mathrm{Ne} / \mathrm{ERN}$, the earlier the latency of the P1 for angry faces (see Figure 5).

\section{DISCUSSION}

The present experiment investigated the effect of instructing a focus of attention towards external signals on the neural correlates of performance monitoring. Angry and neutral faces were presented after each response in a response-choice task. The expression of the faces was linked to the accuracy of the response, but only half of the participants were explicitly instructed about a possible relationship. When the instruction indicated a relationship between task performance and face stimulus, the Ne/ERN to erroneous responses was reduced. This reduction could reflect an instruction-dependent reallocation of monitoring resources from internal to external signals. In agreement with such an interpretation, the N170 to subsequent face stimuli was enhanced in the instructed compared to the not-instructed group, but instructions did not affect the amplitude of the FRN. The present results, hence, point to the possibility that the processing of internal signals can be reduced by focusing on external signals for performance evaluation. Interestingly, we also found a correlation between $\triangle \mathrm{Ne} / \mathrm{ERN}$ and P1-latency benefit for angry faces presented after errors compared to angry faces presented after a correct response. This result corroborates

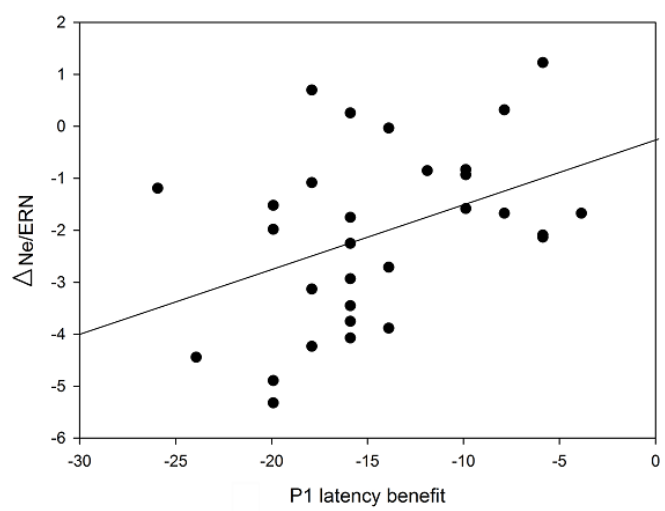

FIGURE 5.

Linear correlation between amplitude of the Ne/ERN-CRN difference and P1 benefit in latency for angry faces after errors compared to angry faces presented after a correct response. 
previous studies on affective priming and suggests a possible negative affective connotation of errors that primes the processing of subsequent negative expressions.

Directing the focus of performance monitoring to an external signal by instruction significantly reduced the amplitude of the $\mathrm{Ne} /$ ERN, but it did not affect the magnitude of the other response-related ERP components of performance evaluation, like different $\mathrm{Pe} / \mathrm{Pc}$ subcomponents. Importantly, participants in the two experimental groups did not differ in their performance, both in the response-choice task and the recognition task, and in their punishment/reward sensitivity, as measured by the BIS/BAS scales. This limits the interpretations of the obtained Ne/ERN modulations in terms of between-groups differences in performance (Gehring et al., 1993; Luu, Flaisch, \& Tucker, 2000) and personality (Boksem et al., 2008). Moreover, the relationship between feedback and performance was similar for all participants and the stimulus-response mapping was clearly stated. Hence, the smaller $\mathrm{Ne} / \mathrm{ERN}$ in the instructed group can be seen as a specific instructiondependent modulation of performance monitoring. In other words, linking an emotional face to performance is sufficient to reduce the monitoring of internal signals, irrespective of the validity of the feedback for accurate performance evaluation.

The observed modulation of the Ne/ERN between groups fits well with the assumption that the processing of internally generated information is reduced when the instruction suggests that an external signal can be used to evaluate the accuracy of the response. Ogawa et al. (2011) found a reduced Ne/ERN in a context of verbal admonishment and suggested that the focus of performance monitoring is reallocated from internal to external signals when an external signal is offered for performance evaluation. In line with this argument, we also found a larger N170 to face stimuli in the instructed group, indicating that participants in this group might have allocated enhanced attention to the external face feedback (Holmes et al., 2003). Taken together, the modulations of the Ne/ERN and the N170 in opposite directions are in line with the assumption that the instructed group reallocated the focus of performance monitoring from internal to external signals.

The present findings suggest that the focus of performance monitoring can be shifted from internal to external signals by explicit instruction even when the information content of the external signal is the same for all participants. Most of the studies in the field of performance monitoring reported switches from an internal to an external focus dependent on feedback information content (Holroyd \& Coles, 2002; Nieuwenhuis et al., 2002) or feedback reliability (Walentowska et al., 2016). In the present design, however, the external signal was a correct feedback to performance for only $66.6 \%$ of the errors and the criteria for response evaluation were clearly defined and easily evaluable. Consequently, internal signals should have been prioritised, regardless of any possible relationship between external signals and performance. In contrast, more impact seems to be given to the external signal according to the instruction. We argue that participants in the instructed group could have preferentially attended to face stimuli in order to understand the before-hand instructed relationship, whereas participants in the not-instructed group simply accepted the absence of a clear relationship between performance and face stimuli and preferentially focused on internal signals. Unfortunately, a performance at ceiling in the recognition task did not allow us to further explore whether the allocation of performance monitoring on the external stimulus determined any behavioural benefit. In addition, being instructed about a relationship between performance and external signal, or looking for such relationship, did not boost participants' awareness of it. This suggests that explicit instructions about a relationship affect the dynamics in signal processing for performance monitoring but do not bias the subjective consideration of a $66.6 \%$ contingency between errors and angry faces as valid feedback.

In contrast to the $\mathrm{Ne} / \mathrm{ERN}$, the $\mathrm{CRN}$ was not modulated by instructions, indicating that instructions affected only those processes that are specific to the monitoring of errors, and not those general monitoring processes that are common for both correct and incorrect responses. This result constitutes further evidence for different underlying processes of Ne/ERN and CRN (Yordanova, Falkenstein, Hohnsbein, \& Kolev, 2004). Moreover, the absence of any significant effect of instruction on the early and the late Pe suggests that instructions influence early, and probably automatic, information processing, but not those late stages of performance monitoring that generally involve error awareness. On the other hand, instruction-dependent modulations of the N170, in the absence of correspondent FRN modulations, suggest the possibility that face stimuli acquire a particular saliency in the instructed group, even when they are not necessary for appropriate performance monitoring, but this saliency does not affect their interpretation. However, FRN analyses in the present experiment should be treated cautiously because of the concurrent activity of higher emotional processes usually eliciting an early posterior negativity (Rellecke, Sommer, \& Schacht, 2012) in the same time window. Similarly, processes underlying modulations of the positive peak preceding the FRN are debatable (Potts, Martin, Burton, \& Montague, 2006). In fact, face processing evokes both an occipito-temporal negativity, the N170, and a frontal positivity (Jeffreys, 1989), the vertex positive potential (VPP). These two potentials seem associated with the activity of the same neural generator (Joyce \& Rossion, 2005). Therefore, the significant N170 effect might hinder an accurate analysis of FRN effects.

Importantly, only the N170 amplitude was significantly enlarged in the group explicitly instructed about the relationship between performance and facial expression. Looking for such a relationship or a more general tendency to pay attention to the face stimuli, hence, altered only the attention-related processes specific to the structural encoding of the face. Strikingly, while main effects of emotion were observed for both the latency of P1 and N170 components, no Emotion $\times$ Group interactions were present. Two important implications ensue from such findings. First, emotion effects can be considered independent of top-down influences like the group instructions in the present experiment. Second, since modulations of P1 and N170 by emotional expressions can be considered to be a consequence of specific attentional mechanisms (i.e., emotional attention, for a review see Pourtois, Schettino, \& Vuilleumier, 2013), this study suggests that instruction influenced specific face processing stages (e.g., structural encoding), 
whereas emotional attention influences remain unaffected. Notably, P1 and N170 latencies were earlier for angry faces after an error and, again, both were not affected by instruction. We therefore ascribe the N170 latency effect to a carry-over from the latency modulation of the P1.

Privileged processing of an angry facial expression after an error was evident already at early levels of visual stimulus processing, preceding the N170. The P1, reflecting early endogenous allocation of attention, peaked earlier and was larger in amplitude for angry faces that followed an incorrect response as compared to all other conditions. This processing benefit could reflect affective priming following erroneous responses (Aarts et al., 2012, 2013). Interestingly, the correlation between P1-latency benefit and $\triangle \mathrm{Ne} / \mathrm{ERN}$ further supports affective priming. Processing of angry faces after errors was faster when the difference between Ne/ERN and CRN was larger. The $\triangle \mathrm{Ne} / \mathrm{ERN}$ is often associated with the experience of negative affect (Hajcak \& Foti, 2008; Hajcak, McDonald, \& Simons, 2004; Luu et al., 2000), and it is enhanced in psychological disorders characterized by deep distress for errors, as in anxiety disorder (Proudfit, Inzlicht, \& Mennin, 2013). Thus, the correlation between P1 latency and $\Delta \mathrm{Ne} / \mathrm{ERN}$ might indicate that an erroneous response primes negative affect so that the processing of angry faces got a latency benefit.

Potential limits of the present experiment may include the use of a between-subjects design, with the consequent possibility of Type-I or Type-II errors, and the unequal number of trials between conditions due to the high number of correct responses. Regarding the first limitation, questionnaires and behavioural performance measures did not indicate any significant differences between groups in the present study and the opposite effects of Ne/ERN and N170 amplitudes were predicted between-subjects effects, emphasising the robustness of the present findings. Potential differences in signal-to-noise ratios across conditions might affect within-subject contrasts but not the contrasts between groups. Therefore, the reported effects of instruction on the amplitude of the Ne/ERN and the N170 are both reliable, whereas contrasts between conditions should be considered with caution. The issues of unequal trial numbers between conditions and the consequent potential differences in signal-to-noise ratios do not apply to the correlation, because an equal number of angry faces followed correct and incorrect responses. The significant correlation between internal performance monitoring and facilitated perceptual processes for angry faces is an electrophysiological corroboration of the behavioural finding that errors prime the processing of subsequent negative material.

The outcome of the present investigation opens a wide field for future research on performance monitoring dynamics. In addition, the present findings might offer a new approach to psychological disorders characterized by pathological internal performance monitoring (Olvet \& Hajcak, 2008). Internalisation disorders are characterised by the inclination to an inward expression of distress and a hyperactive monitoring system. An increased Ne/ERN is present in psychological disorders characterised by internalisation, like obsessive-compulsive disorder (Gehring, Himle, \& Nisenson, 2000; Johannes et al., 2001; Ruchsow et al., 2005), general anxiety disorder (Weinberg, Olvet,
\& Hajcak, 2010), and depression (Chiu \& Deldin, 2007; Holmes \& Pizzagalli, 2008). Whether patients with internalisation disorders show a normal flexibility to move from internal to external signals according to instruction, like participants in the present experiment, should be addressed by future research.

To conclude, this study shows that (a) internal monitoring can be reduced by instruction suggesting the processing of external stimuli, leading to enhanced processing of the feedback, and (b) that the negative feelings associated with errors, indexed by the $\Delta \mathrm{Ne} / \mathrm{ERN}$, determine facilitation in processing feedback stimuli with a negative valence. In addition, the observed instruction-dependent effects on performance monitoring represent a promising opportunity for the study of performance monitoring dynamics both in healthy and clinical populations.

\section{ACKNOWLEDGMENTS}

This research was supported by funding of the Deutsche Forschungsgemeinschaft (STU 248/3-2). We are grateful to Dorit Wenke and Anne Löffler for their cooperation in developing a highly demanding interference task and to Werner Sommer and Andrea Hildebrandt for supporting us with numerous well-controlled face stimuli. We thank Christopher Saville for proofreading. Moreover, we thank Anna Weber for her assistance in data collection.

\section{REFERENCES}

Aarts, K., De Houwer, J., \& Pourtois, G. (2012). Evidence for the automatic evaluation of self-generated actions. Cognition, 124, 117-127. doi: 10.1016/j.cognition.2012.05.009

Aarts, K., De Houwer, J., \& Pourtois, G. (2013). Erroneous and correct actions have a different affective valence: Evidence from ERPs. Emotion, 13, 960-973. doi: 10.1037/a0032808 wWw

Aarts, K., \& Pourtois, G. (2012). Anxiety disrupts the evaluative component of performance monitoring: An ERP study. Neuropsychologia, 50, 1286-1296. doi: 10.1016/j. neuropsychologia.2012.02.012 $\overline{\underline{\mathrm{WW}}}$

Arbel, Y., \& Donchin, E. (2009). Parsing the componential structure of post-error ERPs: A principal component analysis of ERPs following errors. Psychophysiology, 46, 1179-1189. doi:

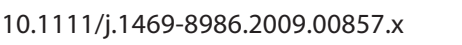

Bellebaum, C., \& Colosio, M. (2014). From feedback- to responsebased performance monitoring in active and observational learning. Journal of Cognitive Neuroscience, 26, 2111-2127. doi: 10.1162/jocn_a_00612 $\underline{\mathrm{WWW}}$

Blair, R. J. (2003). Facial expressions, their communicatory functions and neuro-cognitive substrates. Philosophical Transactions of the Royal Society of London B: Biological Sciences, 358, 561-572. doi: 10.1098/rstb.2002.1220 |wWw

Boksem, M. A., Ruys, K. I., \& Aarts, H. (2011). Facing disapproval: Performance monitoring in a social context. SocialNeuroscience, 6, 360-368. doi: 10.1080/17470919.2011.556813 |

Boksem, M. A., Tops, M., Kostermans, E., \& De Cremer, D. (2008). Sensitivity to punishment and reward omission: Evidence 
from error-related ERP components. Biological Psychology, 79, 185-192. doi: 10.1016/j.biopsycho.2008.04.010 WwW

Boldt, A., \& Yeung, N. (2015). Shared neural markers of decision confidence and error detection. Journal of Neuroscience, 35, 3478-3484. doi: 10.1523/JNEUROSCI.0797-14.2015 Ww

Carver, C. S., \& White, T. L. (1994). Behavioral inhibition, behavioral activation, and affective responses to impending reward and punishment: The BIS/BAS scales. Journal of Personality and Social Psychology, 67, 319-333. doi: 10.1037/0022-3514 67.2.319

Chiu, P. H., \& Deldin, P. J. (2007). Neural evidence for enhanced error detection in major depressive disorder. The American Journal of Psychiatry, 164, 608-616. doi: 10.1176/ajp.2007.164.4.608 [WW

Ebner, N. C., Riediger, M., \& Lindenberger, U. (2010). FACES-a database of facial expressions in young, middle-aged, and older women and men: Development and validation. Behavior Research Methods, 42, 351-362. doi: 10.3758/BRM.42.1.351 WWW

Eriksen, B. A., \& Eriksen, C. W. (1974). Effects of noise letters upon the identification of a target letter in a nonsearch task. Perception \& Psychophysics, 16, 143-149. doi: 10.3758/ BF03203267

Falkenstein, M., Hohnsbein, J., Hoormann, J., \& Blanke, L. (1991). Effects of crossmodal divided attention on late ERP components. II. Error processing in choice reaction tasks. Electroencephalography and Clinical Neurophysiology, 78, 447-455. doi: 10.1016/0013-4694(91)90062-9 WWW

Gehring, W. J., Goss, B., Coles, M. G. H., Meyer, D. E., \& Donchin, E. (1993). A neural system for error detection and compensation. Psychological Science, 4, 385-390. doi: 10.1111/j.14679280.1993.tb00586.x

Gehring, W. J., Himle, J., \& Nisenson, L. G. (2000). Action-monitoring dysfunction in obsessive-compulsive disorder. Psychological Science, 11, 1-6. doi: 10.1111/1467-9280.00206 WWW

Gentsch, K., Grandjean, D., \& Scherer, K. R. (2013). Temporal dynamics of event-related potentials related to goal conduciveness and power appraisals. Psychophysiology, 50, 1010-1022. doi: $10.1111 /$ psyp. $12079 \underline{\underline{W W}}$

Gibbons, H., Schnuerch, R., \& Stahl, J. (2016). From positivity to negativity bias: Ambiguity affects the neurophysiological signatures of feedback processing. Journal of Cognitive

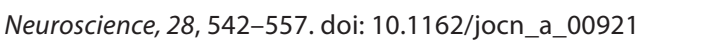

Hajcak, G., \& Foti, D. (2008). Errors are aversive: Defensive motivation and the error-related negativity. Psychological Science, 19, 103-108. doi: 10.1111/j.1467-9280.2008.02053.x WwW

Hajcak, G., McDonald, N., \& Simons, R. F. (2004). Error-related psychophysiology and negative affect. Brain \& Cognition, 56, 189-197. doi: 10.1016/j.bandc.2003.11.001 WWW

Heldmann, M., Rüsseler, J., \& Münte, T. F. (2008). Internal and external information in error processing. BMC Neuroscience, 9, 33. doi: 10.1186/1471-2202-9-33 WWW
Hillyard, S. A., \& Anllo-Vento, L. (1998). Event-related brain potentials in the study of visual selective attention. Proceedings of the National Academy of Sciences of the United States of America, 95, 781-787. $\underline{\underline{W W}}$

Holmes, A. J., \& Pizzagalli, D. A. (2008). Response conflict and frontocingulate dysfunction in unmedicated participants with major depression. Neuropsychologia, 46, 2904-2913. doi: 10.1016/j.neuropsychologia.2008.05.028 WWW

Holmes, A. J., Vuilleumier, P., \& Eimer, M. (2003). The processing of emotional facial expression is gated by spatial attention: Evidence from event-related brain potentials. Cognitive Brain Research, 16, 174-184. doi: 10.1016/S0926-6410(02)00268-9 WWW

Holroyd, C. B., \& Coles, M. G. H. (2002). The neural basis of human error processing: Reinforcement learning, dopamine, and the error-related negativity. Psychological Review, 109, 679-709. doi: 10.1037/0033-295x.109.4.679

Jeffreys, D. A. (1989). A face-responsive potential recorded from the human scalp. Experimental Brain Research, 78, 193-202. doi: 10.1007/BF00230699 $\overline{\text { WWW }}$

Johannes, S., Wieringa, B. M., Nager, W., Rada, D., Dengler, R., Emrich, H. M., . . Dietrich, D. E. (2001). Discrepant target detection and action monitoring in obsessive-compulsive disorder. Psychiatry Research, 108, 101-110. doi: 10.1016/S09254927(01)00117-2 WWW

Joyce, C., \& Rossion, B. (2005). The face-sensitive N170 and VPP components manifest the same brain processes: The effect of reference electrode site. Clinical Neurophysiology, 116, 2613-

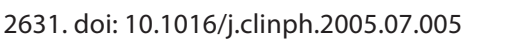

Klinnert, M. D., Campos, J. J., Sorce, J. F., Emde, R. N., \& Svejda, M. (1983). Emotions as behavior regulators: Social referencing in infancy. In R. Plutchik \& H. Kellerman (Eds.), Emotions: Theory, research and experience (Vol. 2, pp. 57-86). New York, NY: Academic Press.

Luu, P., Flaisch, T., \& Tucker, D. M. (2000). Medial frontal cortex in action monitoring. Journal of Neuroscience, 20, 464-469.

Mangun, G. R., \& Hillyard, S. A. (1991). Modulations of sensory-evoked brain potentials indicate changes in perceptual processing during visual-spatial priming. Journal of Experimental Psychology: Human Perception \& Performance, 17,

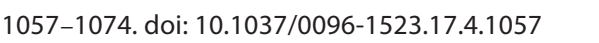

Moses, L. J., Baldwin, D. A., Rosicky, J. G., \& Tidball, G. (2001). Evidence for referential understanding in the emotions domain at twelve and eighteen months. Child Development, 72, 718-735. doi: 10.1111/1467-8624.00311 ww

Müller, S. V., Möller, J., Rodriguez-Fornells, A., \& Münte, T. F. (2005). Brain potentials related to self-generated and external information used for performance monitoring. Clinical Neurophysiology, 116, 63-74. doi: 10.1016/j.clinph.2004.07.009 WWW

Nieuwenhuis, S., Ridderinkhof, K. R., Talsma, D., Coles, M. G., Holroyd, C. B., Kok, A., \& van der Molen, M. W. (2002). A com- 
putational account of altered error processing in older age: Dopamine and the error-related negativity. Cognitive, Affective, \& Behavioral Neuroscience, 2, 19-36. doi: 10.3758/CABN.2.1.19 WWW

Nieuwenhuis, S., Yeung, N., Holroyd, C. B., Schurger, A., \& Cohen, J. D. (2004). Sensitivity of electrophysiological activity from medial frontal cortex to utilitarian and performance feedback. Cerebral Cortex, 14, 741-747. doi: 10.1093/cercor/bhh034 WwW

Ogawa, K., Masaki, H., Yamazaki, K., \& Sommer, W. (2011). The influence of emotions due to verbal admonishment and encouragement on performance monitoring. Neuroreport, 22, 313-318. doi: 10.1097/WNR.0b013e328345bf42 |WWW

Oldfield, R. C. (1971). The assessment and analysis of handedness: The Edinburgh inventory. Neuropsychologia, 9, 97-113. doi: 10.1016/0028-3932(71)90067-4 |WWW

Olvet, D. M., \& Hajcak, G. (2008). The error-related negativity (ERN) and psychopathology: Toward an endophenotype. Clinical Psychology Review, 28, 1343-1354. doi: 10.1016/j. cpr.2008.07.003 WWW

Olvet, D. M., \& Hajcak, G. (2009a). The effect of trial-to-trial feedback on the error-related negativity and its relationship with anxiety. Cognitive, Affective, \& Behavioral Neuroscience, 9, 427-433. doi: 10.3758/CABN.9.4.427 WWW

Olvet, D. M., \& Hajcak, G. (2009b). The stability of error-related brain activity with increasing trials. Psychophysiology, 46,

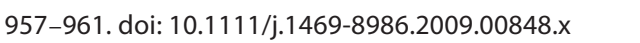

Pontifex, M. B., Scudder, M. R., Brown, M. L., O'Leary, K. C., Wu, C. T., Themanson, J. R., \& Hillman, C. H. (2010). On the number of trials necessary for stabilization of error-related brain activity across the life span. Psychophysiology, 47, 767-773. doi: 10.1111/j.1469-8986.2010.00974.x WW

Potts, G. F., Martin, L. E., Burton, P., \& Montague, P. R. (2006). When things are better or worse than expected: The medial frontal cortex and the allocation of processing resources. Journal of Cognitive Neuroscience, 18, 1112-1119. doi: 10.1162/ jocn.2006.18.7.1112 WWW

Pourtois, G., Schettino, A., \& Vuilleumier, P. (2013). Brain mechanisms for emotional influences on perception and attention: What is magic and what is not. Biological Psychology, 92, 492-512. doi: 10.1016/j.biopsycho.2012.02.007 |wWW

Proudfit, G. H., Inzlicht, M., \& Mennin, D. S. (2013). Anxiety and error monitoring: The importance of motivation and emotion. Frontiers in Human Neuroscience, 7:636. doi: 10.3389/ fnhum.2013.00636 WWW

Rabbitt, P. M. (1966). Errors and error correction in choice-response tasks. Journal of Experimental Psychology, 71, 264-272. doi: $10.1037 / \mathrm{h} 0022853 \underline{\mathrm{WWW}}$

Rellecke, J., Sommer, W., \& Schacht, A. (2012). Does processing of emotional facial expressions depend on intention? Time-resolved evidence from event-related brain potentials. Biological Psychology, 90, 23-32. doi: 10.1016/j.

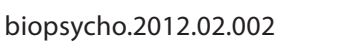

Repacholi, B. M. (1998). Infants' use of attentional cues to identify the referent of another person's emotional expression. Developmental Psychology, 34, 1017-1025. doi: 10.1037/00121649.34.5.1017 WWW

Ruchsow, M., Gron, G., Reuter, K., Spitzer, M., Hermle, L., \& Kiefer, M. (2005). Error-related brain activity in patients with obsessive-compulsive disorder and in healthy controls. Journal of Psychophysiology, 19, 298-304. doi: 10.1027/0269-8803 .19.4.298

Shalgi, S., Barkan, I., \& Deouell, L. Y. (2009). On the positive side of error processing: Error-awareness positivity revisited. European Journal of Neuroscience, 29, 1522-1532. doi: 10.1111/j.14609568.2009.06690.x WWW

Simon, J. R. (1969). Reactions toward the source of stimulation. Journal of Experimental Psychology, 81, 174-176. doi: 10.1037/ h0027448 WW

Sorce, J. F., Emde, R. N., Campos, J. J., \& Klinnert, M. D. (1985). Maternal emotional signaling: Its effect on the visual cliff behavior of 1-year-olds. Developmental Psychology, 21, 195-200. doi: 10.1037/0012-1649.21.1.195

Stahl, J. (2010). Error detection and the use of internal and external error indicators: An investigation of the first-indicator hypothesis. International Journal of Psychophysiology, 77, 43-52.

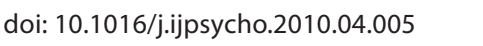

Steinhauser, M., \& Yeung, N. (2012). Error awareness as evidence accumulation: Effects of speed-accuracy trade-off on error signaling. Frontiers in Human Neuroscience, 6:240. doi: 10.3389/ fnhum.2012.00240

Themanson, J. R., Rosen, P. J., Pontifex, M. B., Hillman, C. H., \& McAuley, E. (2012). Alterations in error-related brain activity and post-error behavior over time. Brain \& Cognition, 80, 257-265. doi: 10.1016/j.bandc.2012.07.003 |WWW

Ullsperger, M., Danielmeier, C., \& Jocham, G. (2014). Neurophysiology of performance monitoring and adaptive behavior. Physiological Reviews, 94, 35-79. doi: 10.1152/ physrev.00041.2012 www

Walentowska, W., Moors, A., Paul, K., \& Pourtois, G. (2016). Goal relevance influences performance monitoring at the level of the FRN and P3 components. Psychophysiology, 53, 1020-1033. doi: 10.1111/psyp.12651 WWW

Weinberg, A., Olvet, D. M., \& Hajcak, G. (2010). Increased errorrelated brain activity in generalized anxiety disorder. Biological Psychology, 85, 472-480. doi: 10.1016/j.biopsycho.2010.09.011 WWW

Yeung, N., \& Summerfield, C. (2012). Metacognition in human decision-making: Confidence and error monitoring. Philosophical Transactions of the Royal Society of London B: Biological Sciences,

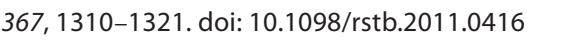

Yordanova, J., Falkenstein, M., Hohnsbein, J., \& Kolev, V. (2004). Parallel systems of error processing in the brain. Neuroimage, 22, 590-602. doi: 10.1016/j.neuroimage.2004.01.040 WWW 